\section{Marc Parmentier Pierre Vanderhaeghen Stéphane Schurmans Frédérick Libert Gilbert Vassart}

\title{
Génétique moléculaire des récepteurs olfactifs
}

Les gènes codant pour les récepteurs olfactifs ont été isolés à partir d'une banque d'ADNc de la muqueuse olfactive. Ils constituent une famille très étendue (plusieurs centaines de gènes) codant pour des récepteurs à sept passages transmembranaires couplés, par l'intermédiaire de protéines G, à l'activation de l'adénylyl cyclase et de la phospholipase C. La grande diversité des récepteurs olfactifs réside surtout dans les domaines transmembranaires III, IV, V et VI, qui portent les sites de liaison des ligands, les molécules odorantes, et répond sans doute à la diversité des ligands. Ces récepteurs sont pratiquement spécifiques de la muqueuse olfactive, mais ont également été détectés dans le testicule, ce qui pose la question d'un rôle possible dans la chémoattraction des spermatozoïdes?

\begin{abstract}
ADRESSES
M. Parmentier : chercheur qualifié du FNRS. P. Vanderhaeghen : aspirant du FNRS. S. Schurmans : aspirant du FNRS. F. Libert : chercheur qualifié du FNRS. IRIBHN, Université Libre de Bruxelles, Campus Erasme 808 , route de Lennik, 1070 Bruxelles, Belgique. G. Vassart : chef du service de génétique medicale. Université Libre de Bruxelles, Campus Erasme 808, route de Lennik, 1070 Bruxelles, Belgique.
\end{abstract}

\section{TIRÉS A PART}

M. Parmentier.

$m / s n^{\circ} 11$, vol. 10, novembre 94 es cellules réceptrices du système olfactif sont des neurones bipolaires localisés au sein d'un épithélium spécialisé recouvrant la paroi dorsale des cavités nasales. Ces neurones envoient un axone unique non ramifié vers le bulbe olfactif homolatéral, à travers la lame criblée de l'ethmoïde, et leur dendrite vers la lumière de la cavité nasale. Au contact de cette cavité, les dendrites se terminent en une protubérance, d'où projettent cinq à vingt cils dans l'épaisse couche de mucus tapissant l'épithélium. Les cils constituent le siège de la réception des signaux olfactifs. Le corps cellulaire du neurone contient, quant à lui, les canaux responsables de la production des potentiels d'action. Les neurones olfactifs possèdent la particularité, unique parmi les neurones de vertébrés supérieurs, de proliférer tout au long de la vie à partir d'une population de cellules souches, et donc de régénérer à la suite de la disparition régulière des cellules différenciées.

Le système olfactif est à même de distinguer plusieurs milliers de substances odorantes. Ce remarquable pouvoir discriminant est dû à l'interaction de ces substances odorantes avec des récepteurs spécifiques portés par les cils des neurones olfactifs. La stimulation des récepteurs olfactifs conduit à la stimulation rapide de l'adénylyl cyclase ou de la phospholipase C, en fonction de la structure chimique de la molécule [1-3]. L'élévation du taux d'AMP cyclique intracellulaire (AMPc) conduit à l'ouverture d'un canal cationique contrôlé par les nucléotides cycliques [4], et la dépolarisation qui en résulte initie le potentiel d'action. L'accroissement des concentrations d'inositol trisphosphate $\left(\mathrm{IP}_{3}\right)$ dans les neurones olfactifs aurait, par ailleurs, pour effet de contrôler l'ouverture de canaux calciques de la membrane plasmique [5].

Au cours des dernières années, la 


\section{RÉFÉRENCES}

1. Pace U, Hanski E, Salomon Y, Lancet D. Odorant-sensitive adenylate cyclase of olfactory reception. Nature $1985 ; 316: 255-8$.

2. Breer H, Boekhoff I, Tareilus E. Rapid kinetics of second messenger formation in olfactory transduction. Nature $1990 ; 345$ : 65-8.

3. Breer $\mathrm{H}$. Molecular reaction cascades in olfactory signal transduction. J Steroid Biochem Mol Biol 1991 ; 39 : 621-5.

4. Nakamura T, Gold GH. A cyclic nucleotide-gated conductance in olfactory receptor cilia. Nature $1987 ; 325$ : 442-4.

5. Restrepo D, Miyamoto T, Bryant PB Teeter JH. Odor stimuli trigger influx of calcium into olfactory neurons of the channel catfish. Science $1990 ; 249$ : 1166-8.

6. Ronnett GV, Snyder SH. Molecular messengers of olfaction. Trends Neurosci $1992 ; 15: 508-13$.

7. Jones DT, Reed RR. G : an olfactory neuron specific-G protelf involved in odorant signal transduction. Science 1989 ; $244: 790-5$.

8. Bakalyar HA, Reed RR. Identification of a specialized adenylyl cyclase that may mediate odorant detection. Nature 1990; $250: 1403-6$.

9. Dhallan RS, Yau KW, Schrader KA Reed RR. Primary structure and functionnal expression of a cyclic nucleotide-activated channel from olfactory neurons. Nature $1990 ; 347: 184-7$.

10. Buck L, Axel R. A novel multigene family may encode odorant receptors: a molecular basis for odor recognition. Cell $1991 ; 65: 175-87$.

11. Parmentier M, Libert F, Schurmans S, $e t$ al. Members of the olfactory receptor gene family are expressed in mammalian germ cells. Nature $1992 ; 355: 453-5$.

12. Ressler KJ, Sullivan SL, Buck LB. A zonal organization of odorant receptor gene expression in the olfactory epithelium. Cell compréhension des mécanismes moléculaires [6] qui sont à la base de la perception olfactive a fait des progrès considérables (figure 1). On a assisté notamment à l'identification, puis au clonage moléculaire des principales protéines impliquées dans la transduction des signaux olfactifs, telles que la protéine trimérique liant le GTP $\left(\mathrm{G}_{\text {off }}\right)$ [7], l'adénylyl cyclase de type III, spécifique des neurones olfactifs [8] et le canal ionique contrôlé par les nucléotides cycliques [9]. Une famille de gènes codant pour des récepteurs olfactifs potentiels a enfin été clonée; d'abord chez le rat [10], puis chez le chien et l'homme [11], la souris [12] et le poisson-chat [13]. Un facteur de transcription, Olf- 1 , impliqué dans le contrôle de l'expression de plusieurs gènes, exprimé spécifiquement dans les neurones olfactifs, a également été caractérisé [14]. On connaît mal, en revanche, le rôle de l'olfactory binding protein (OBP), qui représente à elle seule environ $1 \%$ des protéines solubles du mucus nasal, et qui lie avec une faible affi- nité pratiquement tous les odorants liposolubles [15]. Il est possible que cette protéine soit impliquée dans la concentration ou le transport des molécules odorantes au sein de la couche de mucus, voire dans la présentation de ces molécules aux récepteurs olfactifs eux-mêmes. La figure 1 présente la conception actuelle de la transduction des signaux olfactifs dans les neurones spécialisés de la muqueuse nasale.

\section{Clonage des gènes des récepteurs olfactifs}

Les gènes des récepteurs olfactifs ont tout d'abord été clonés par Buck et Axel [10] à partir d'ARN de muqueuse olfactive de rat, en utilisant la technique d'amplification en chaîne par la polymérase (PCR) et des amorces dégénérées correspondant aux segments les plus conservés parmi les récepteurs couplés aux protéines $G$ [16]. La sélection des produits d'amplification reposait sur l'hypothèse que les gènes codant pour les récepteurs olfactifs devaient

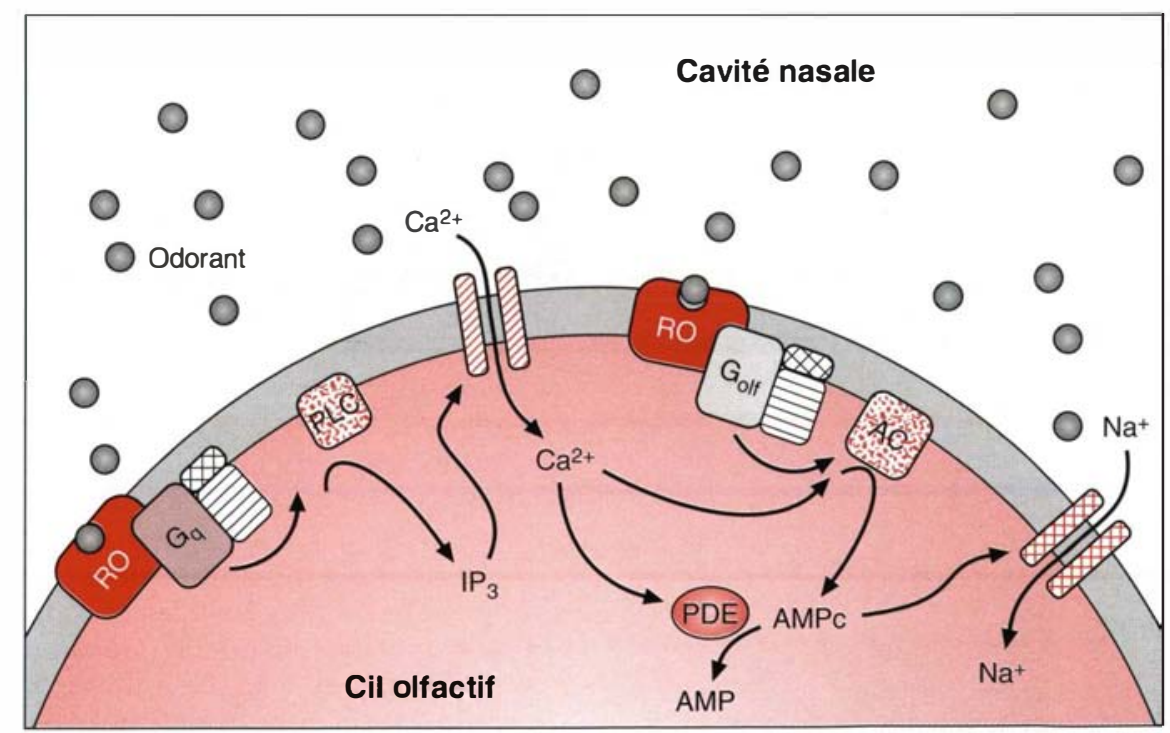

Figure 1. Transduction des signaux dans les cils des neurones olfactifs de la muqueuse nasale. Après liaison des molécules odorantes, les récepteurs olfactifs (RO) activent l'adénylyl cyclase (AC) ou la phospholipase $C$ (PLC) par l'intermédiaire des protéines $G$ trimériques $(\alpha \beta \gamma) G_{\text {olf }}$ et $G_{q^{*}} L^{\prime}$ 'augmentation de l'AMPc intracellulaire aboutit à la dépolarisation cellulaire par l'ouverture d'un canal sodium. Contrairement au schéma classique du calcium libéré à partir de réserves intracellulaires, il semble que, dans les cils olfactifs, I'IP ${ }_{3}$ provoque une entrée de calcium extracellulaire [6]. L'action du calcium intracellulaire consiste en une stimulation de l'activité de la phosphodiestérase (PDE) et peut-être de l'adénylyl cyclase (AC). 
être exprimés exclusivement dans les neurones olfactifs, et que ces gènes devaient constituer une grande famille de gènes homologues. Des clones répondant à ces critères furent isolés, et les séquences codantes complètes de certains gènes isolés d'une banque d'ADN complémentaire de muqueuse olfactive. Buck et Axel décrivirent ainsi le clonage de dix récepteurs olfactifs complets, et de huit séquences partielles additionnelles. Utilisant ces données, Levy et al. [17] rapportèrent l'amplification de neuf séquences partielles de rat s'étendant entre les segments transmembranaires II et IV, et présentant plus de diversité que les dix-huit séquences originales de Buck et Axel [10].

Le clonage par homologie utilisant la PCR, a permis dans notre laboratoire de cloner à partir d'ADN génomique ou d'ADN complémentaire, les gènes codant pour une série de nouveaux récepteurs couplés aux protéines G [16, 18]. Parmi les gènes codant pour ces récepteurs non identifiés (et qualifiés d'orphelins), un clone génomique (HGMP07) fut caractérisé par une expression limitée au testicule, et son appartenance à une grande sous-famille de gènes présentant des homologies de séquences importantes. Des clones complets codant pour seize récepteurs apparentés à HGMP07 ont été isolés de banques testiculaires et génomiques humaines [11]. La comparaison de ces séquences à celles publiées entre-temps par Buck et Axel [10] démontra, de plus, que les protéines codées par cette famille de gènes représentaient l'équivalent humain des récepteurs olfactifs potentiels de rat. Des séquences partielles additionnelles furent ensuite obtenues par PCR à partir d'ADN génomique et d'ADNc, chez l'homme et chez le chien. Un clone complet, codant pour un récepteur appelé DTMT, dont les transcrits sont particulièrement abondants dans le testicule de chien, fut isolé à partir d'une banque génomique de chien [11]. D'autres gènes codant pour des membres de la famille des récepteurs olfactifs ont ultérieurement été clonés chez la souris [19, 12], ainsi que chez le poisson-chat [13].
Plusieurs équipes ont commencé à déterminer l'organisation de la famille de gènes codant pour les récepteurs olfactifs dans le génome humain. Des résultats préliminaires indiquent que ces gènes ne sont pas distribués dans l'ensemble du génome, mais regroupés en un nombre restreint de localisations. Nous avons ainsi localisé un de ces gènes ( HGMPO7E, codant pour une protéine représentée dans la figure $2 A$ ) dans la région 17 pl3 du génome humain (figure 2B) [20]. D'autres gènes structurellement apparentés semblent localisés dans la même région, ainsi que sur le segment 3pl3-3p21. D’autres gènes olfactifs sont également localisés sur ce même chromosome 17 [21], ainsi que sur le chromosome 19 [22].

\section{Taille de la famille multigénique}

La taille de la famille multigénique qui code pour les récepteurs olfactifs a été estimée sur la base des résutats d'hybridation de Southern blots génomiques, et de criblages de librairies génomiques, en utilisant les sondes disponibles. Buck et Axel [10] ont ainsi estimé que cent à deux cents gènes par génome haploïde représentait un nombre minimal chez le rat. Notre propre estimation pour l'homme et pour le chien est de quatre cents gènes au moins [11]. Les chiffres d'un millier de gènes pour les mammifères et d'une centaine pour les poissons [13] sont généralement admis. D'un point de vue structural, les récepteurs olf actifs peuvent être divisés en sous-familles (figure 3), mais rien n'indique actuellement que cette classification anatomique puisse être corrélée à l'interaction de ces récepteurs avec des classes d'odorants, et/ou avec des cascades intracellulaires différentes.

Des fragments génomiques portant plusieurs gènes de récepteurs olfactifs apparentés, organisés en tandems, ont été isolés, renforçant l'hypothèse, mentionnée ci-dessus, d'un regroupement important des gènes homologues dans le génome. L'évolution de cette famille pourrait dès lors impliquer des phénomènes tels que crossing-over inégal et conversion génique, conduisant à une expansion et à une diversification progressive de la famille [10]. Un certain nombre de pseudogènes de récepteurs olfactifs ont également été isolés du génome humain ([23] et nos résultats non publiés). Étant donné le rôle relativement modeste joué par le sens olfactif chez l'homme, la pression évolutive pour maintenir une gamme complète de récepteurs fonctionnels est vraisemblablement basse, et une partie du répertoire génique pourrait dès lors avoir été muté en pseudogènes non fonctionnels. L'hypothèse selon laquelle certains gènes sont inactifs, et que des variants alléliques existent dans la population humaine est soutenue par la démonstration du déterminisme génétique du seuil de détection individuel pour des molécules odorantes [24].

\section{Spécificité cellulaire de l'expression}

Sur la base d'expériences de Northern blot, Buck et Axel [10] ont conclu que les récepteurs olfactifs sont exprimés spécifiquement dans les neurones de la muqueuse olfactive. En l'absence de données fonctionnelles, cette distribution constituait en fait un des arguments majeurs en faveur de la nature olfactive de ces récepteurs. Des résultats d'immunohistochimie ont démontré que les neurones olfactifs exprimant un récepteur olfactif spécifique sont distribués largement au sein de la muqueuse [22]. La distribution de différents récepteurs dans la muqueuse olfactive a également été étudiée par hybridation in situ chez le poisson-chat, le rat et la souris. Chez le poisson (où le nombre de récepteurs est estimé à quelques dizaines), chaque récepteur est exprimé dans un petit nombre de cellules, largement distribuées dans l'ensemble de la muqueuse [26]. Chez les rongeurs, la situation est quelque peu différente : la muqueuse olfactive peut être divisée en trois régions (supérieure, médiane et inférieure) s'étendant d'avant en arrière ; il semble que chaque récepteur ne soit exprimé que dans une seule de ces régions et que la distribution des cellules exprimant un récepteur soit aléatoire au sein de cette région $[12,27]$. La muqueuse olfactive des poissons est donc 


\section{RÉFÉRENCES}

13. Ngai J, Dowling MM, Buck L, Axel R, Chess A. The family of genes encoding odorant receptors in the channel catfish. Cell $1993 ; 72: 657-66$.

14. Wang MM, Reed RR. Molecular cloning of the olfactory neuronal transcription factor Olf-1 by genetic selection in yeast. Nature $1993 ; 364: 121-6$.

15. Pevsner J, Sklar PB, Snyder SH. Odorant-binding protein localization to nasal glands and secretions. Proc Natl Acad Sci USA $1986 ; 83: 4942-6$.

16. Libert F, Parmentier M, Lefort A, et al. Selective amplification and cloning of four new members of the $G$ protein-coupled receptor family. Science $1989 ; 244: 569-72$.

17. Levy NS, Bakalyar HA, Reed RR. Signal transduction in olfactory neurons. J Steroid Mol Biol 1991 ; 39 : 633-7.

18. Parmentier M, Libert F, Maenhaut C, et al. Molecular cloning of the thyrotropin receptor. Science $1989 ; 246: 1620-2$.

19. Nef P, Hermans-Borgmeyer I, ArtieresPin $\mathrm{H}$, Beasley L, Dionne VE, Heinemann SF. Spatial pattern of receptor expression in the olfactory epithelium. Proc Nall Acad Sci USA $1992 ; 89$ : 8948-52.

20. Schurmans S, Passage E, Miot F Mattei MG, Vassart G, Parmentier M. The HGMP07E gene encoding a putative olfactory receptor maps to the $17 \mathrm{p} 12-17 \mathrm{pl} 3$

region of the human genome and reveals a MspI restriction fragment length polymorphism. Cytogenet Cell Genet 1993 ; $63: 200-4$.

21. Ben-Arie N, North M, Khen M, Margalit $T$, Lehrach $H$, Lancet $D$. Mapping the olfactory receptor " sub-genome ": implications to human sensory polymorphisms. Proceedings of the Cold Spring Harbor meeting on Genome Mapping and Sequencing. $1992: 280 \mathrm{p}$

22. Reed RR. Mechanisms of sensitivity and specificity in olfaction. Cold Spring Harbor Symposium on Quantitative Biology, Vol. LVII. Cold Spring Harbor Laboratory Press : 1992: 501-4.

23. Selbie LA, Townsend-Nicholson A Iismaa TP, Shine J. Novel G proteincoupled receptors: a gene family of putative human olfactory receptor sequences. Mol constituée d'une seule unité fonctionnelle, alors que la muqueuse des rongeurs comporte trois unités fonctionnelles indépendantes. Dans tous les cas, cependant, il a été estimé que chaque neurone olfactif n'exprime qu'un ou un très petit nombre de récepteurs olfactifs différents. En ce qui concerne l'intégration des signaux en aval des neurones olfactifs, l'hypothèse la plus probable actuellement est que tous les axones originaires de neurones olfactifs exprimant le même récepteur convergent vers un glomérule unique au sein du bulbe olfactif. Cela pose, bien évidemment, le problème du déterminisme réciproque entre l'expression d'un récepteur particulier et l'établissement de la projection centrale du neurone. Il ressort d'études du développement du système olfactif [28] que la connexion des neurones olfactifs avec le bulbe s'effectue avant l'expression des récepteurs. Ce serait donc le contact synaptique qui déterminerait la nature du récepteur exprimé dans chaque neurone. La nature du message susceptible de transmettre cette information est cependant totalement inconnue.

Nous avons signalé plus haut l'expression de certains récepteurs olfactifs dans le testicule de chien [11]. Nous décrivons dans un autre article les conclusions de l'étude de distribution de ces récepteurs testiculaires, ainsi que la démonstration de la présence de la protéine correspondante sur la membrane des spermatozoïdes mûrs [29]. Nos conclusions dans ce cadre sont que certains récepteurs appartenant à la famille olfactive (mais ne constituant pas une sous-famille structurale) sont exprimés préférentiellement dans les cellules germinales mâles, et peu ou pas dans la muqueuse olfactive ellemême. Les relations possibles de cette distribution particulière avec les phénomènes de chémoattraction des spermatozoïdes chez les mammifères restent à démontrer.

Nef $e t$ al. [19] ont étudié la distribution des transcrits du récepteur murin OR3 par Northern blot et hybridation in situ. A partir du douzième jour de la vie embryonnaire, les transcrits sont localisés exclusivement dans une sous-population de cellules de la muqueuse olfactive.
Cependant, aux jours 10 et 11 de la vie embryonnaire, ils décrivent des transcrits OR3 dans des cellules adjacentes à la paroi des vésicules télencéphaliques, le primordium, et les bulbes olf actifs. Les auteurs suggèrent que les cellules exprimant le récepteur OR3 pourraient migrer du système nerveux central vers l'épithélium olf actif. Une telle distribution cérébrale, qui constitue actuellement une observation isolée, devra être recherchée pour d'autres récepteurs olfactifs.

\section{Structure moléculaire}

Tous les gènes de récepteurs olfactifs clonés à ce jour codent pour des protéines de 310 à 333 acides aminés, et de poids moléculaire calculé s'étageant entre 34 et $38 \mathrm{kDa}$. Ces récepteurs possèdent des caractéristiques structurales indépendantes de leur espèce d'origine. Le pourcentage d'identité entre deux récepteurs olfactifs varie de 38 à plus de $90 \%$. L'identité entre les récepteurs olfactifs et d'autres récepteurs couplés aux protéines $G$ ne dépasse pas $30 \%$, montrant que les récepteurs olfactifs constituent bien une sousfamille bien définie des récepteurs à sept domaines transmembranaires (figure 3). Au sein de cette sousfamille, la conservation est la plus grande dans les deuxième, sixième et septième segments transmembranaires, particulièrement à leur jonction avec les boucles intracellulaires. Les premier, troisième, quatrième et cinquième segments transmembranaires sont moins bien conservés. La troisième boucle intracellulaire, qui est connue pour être responsable de l'interaction avec les protéines $G$ dans d'autres récepteurs, n'est pas particulièrement bien conservée par rapport à d'autres domaines de la protéine. Il a été proposé [12] que la faible conservation des domaines transmembranaires III, IV, V et VI (qui constituent ensemble le domaine de liaison dans les récepteurs adrénergiques) pouvait être mise en rapport avec la diversité structurale des ligands, qui caractérise cette famille de récepteurs. La variabilité inhabituelle de ces régions dans les récepteurs du poisson-chat suggère même l'existence d'une pression évolutive favorisant les mutations intervenant 


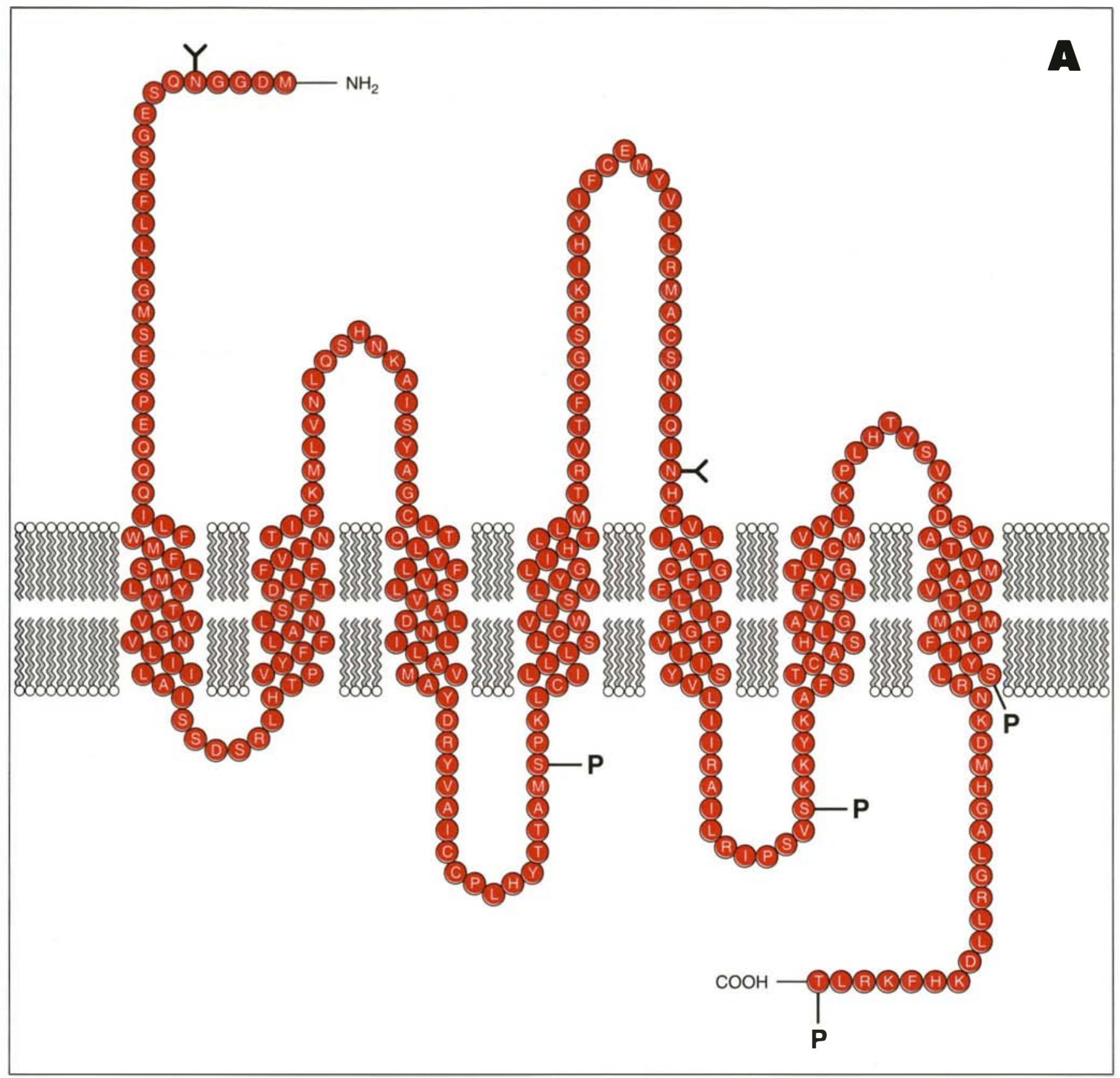

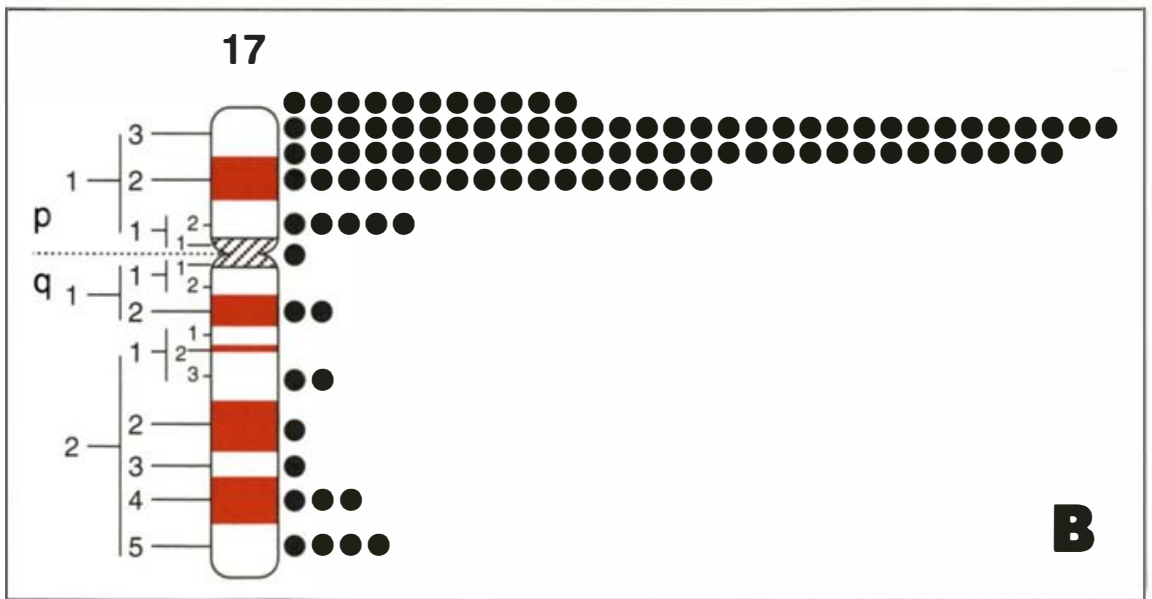

$\mathrm{m} / \mathrm{s} n^{\circ} 11$, vol. 10, novembre 94
Figure 2. A: Organisation transmembranaire probable du récepteur offactif humain HGMPO7E, et localisation des éléments potentiels de régulation fonctionnelle. Les sites potentiels de glycosylation dans le domaine N-terminal et la deuxième boucle extracellulaire sont indiqués (Y), de même que les sites probables de phosphorylation $(P)$ par la protéine kinase $C$ et la protéine kinase dépendante de l'AMPc, dans les deuxième et troisième boucles intracellulaires et le domaine $C$-terminal. B : Localisation du gène du récepteur olfactif HGMPOTE sur le bras court du chromosome 17 humain, déterminée par hybridation in situ [20]. 


\section{RÉFÉRENCES}

24. Gross-Isseroff R, Ophir D, Bartana A Voet $H$, Lancet $D$. Evidence for genetic determination in human twins of olfactory tresholds for a standard odorant. Neurose Lett 1992 ; 141 : 115-8.

25. Koshimoto H, Katoh K, Yoshihara Y, Mori K. Distribution of putative odour receptor proteins in olfactory epithelium. NeuroReport $1992 ; 3: 521-3$.

26. Ngai J, Chess A, Dowling MM, Necles N, Macagno ER, Axel R. Coding of olfactory information: topography of odorant receptor expression in the catfish olfactory epithelium. Cell $1993 ; 72: 667-80$.

27. Vassar R, Ngai J, Axel R. Spatial segregation of odorant receptor expression in the mammalian olfactory epithelium. Cell $1993 ; 74$ : 309-18.

28. Margalit T, Lancet D. Expression of olfactory receptor and transduction genes during rat development. Dev Brain Res $1993 ; 73: 7-16$

29. Vanderhaeghen P, Schurmans S, Vassart G, Parmentier M. Les spermatozoides ont ils un nez? médecine/sciences $1994 ; 10: 1136$ 40 .

30. Raming K, Krieger J, Strotmann J, et al. Cloning and expression of odorant receptors. Nature $1993 ; 361$ : 353-6.

31. Dahmen N, Wang HL, Margolis FL. Expression of olfactory receptors in xenopus oocytes. I Neurochem 1992; 58 : $1176-9$.

32. Sicard G, Holley A. Receptor cell responses to odorants: similarities and differences among odorants. Brain Res $1984 ; 292: 283-96$

33. Schultz $P$, Stannek $P$, Voigt $M$, Jakobs KH, Gierschik P. Complementation of formyl peptide receptor-mediated signal transduction in Xenopus oocytes. Biochem J $1992 ; 284: 207-12$

34. Shirley SG, Polak EH, Mather RA Dodd GH. The effect of concanavalin A on the rat electroolfactogram. J Biochem 1987; $245: 175-84$.

35. Dohlmann HG, Thorner J, Caron MG Lefkowitz RJ. Model systems for the study of seven-transmembrane-segment receptors. Annu Rev Biochem 1991 ; 60 : 653-88.

36. Dawson TM, Arriza JL, Jaworski DE, et al. B-adrenergic receptor kinase-2 and $B$ arrestin-2 as mediators of odorant-induced desensitization. Science $1993 ; 259: 825-9$.

37. Abe K, Kusakabe Y, Tanemura K, Emori Y, Arai S. Primary structure and cellspecific expression of a gustatory $G$ proteincoupled receptor related to olfactory

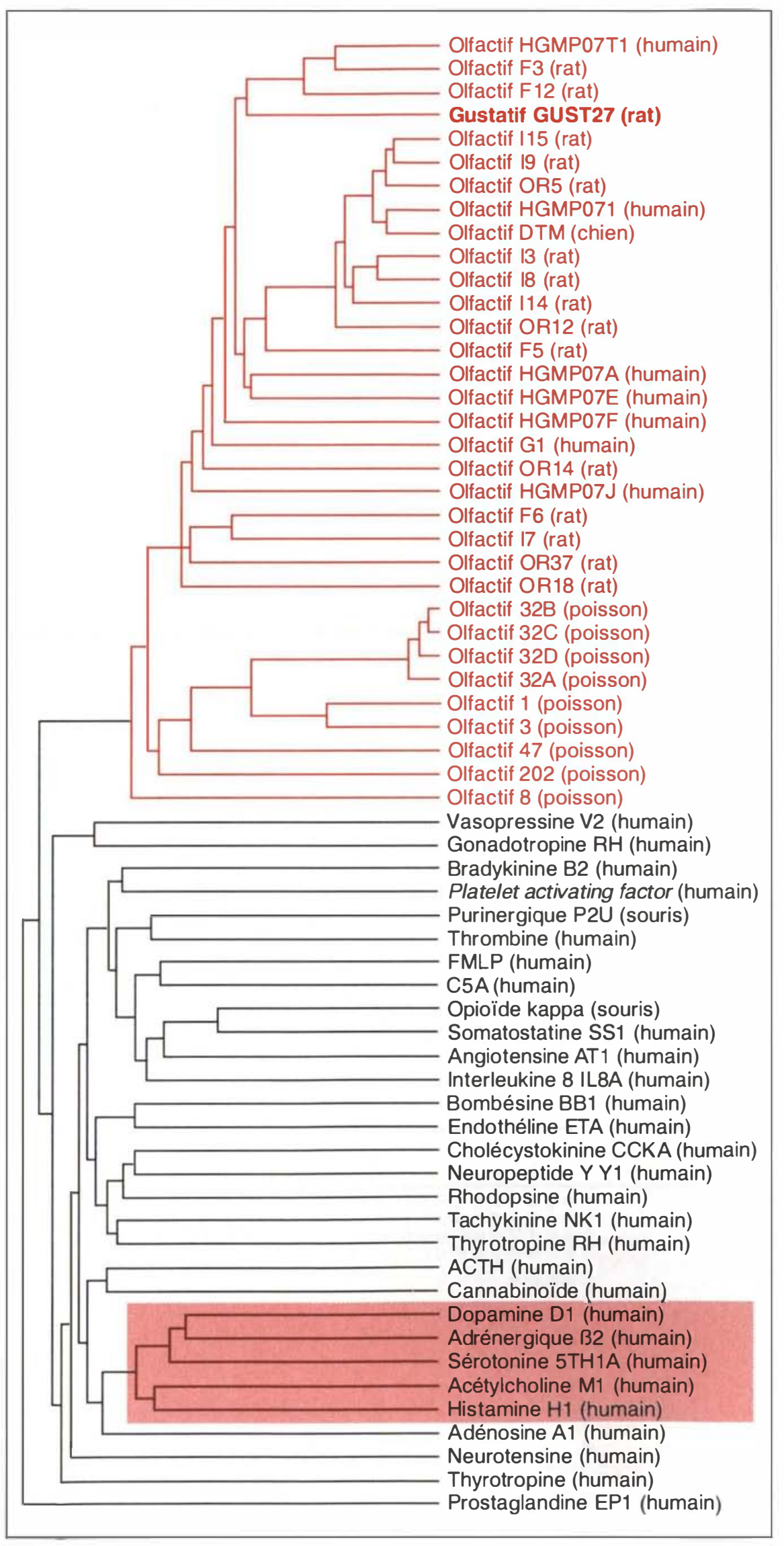

$\mathrm{m} / \mathrm{s} n^{\circ} 11$, vol. 10, novembre 94 
dans les domaines de liaison de ces protéines [13].

F.tant dommé le nombre relativement limité de séquences disponibles, et l'étendue présumée de la famille, il est à présent difficile de déterminer si certains des récepteurs clonés actuellement représentent les homologues d'espèces différentes. Parmi les récepteurs couplés aux protéines $\mathrm{G}$, le pourcentage d'identité entre séquences homologues provenant de mammifères différents varie entre $68 \%$ (récepteurs du C5a du chien et de l'homme) et $98 \%$ (récepteurs cannabinoïdes du rat et de l'homme). Le plus fort pourcentage d'identité entre séquences de récepteurs olfactifs d'espèces différentes dont les gènes ont été clonés est actuellement de $85 \%$ (IDTMT de chien et HGMP07I humain), ce qui est bien dans l'intervalle attendu pour des séquences homologues. Néanmoins, des scores d'identité de plus de $90 \%$ sont rencontrés entre récepteurs isolés de la même espèce, suggérant, soit que des duplications géniques se sont produites après la diversification des espèces de mammifères, soit que des scores supérieurs à $90 \%$ doivent être attendus pour de véritables homologues.

\section{Aspects fonctionnels}

Il est fort probable que la famille de gènes clonés actuellement code effectivement pour tout ou partie des récepteurs olfactifs. Cependant,

Figure 3. Dendrogramme présentant les similarités de séquences entre des récepteurs olfactifs provenant de différentes espèces, et d'autres membres de la famille des récepteurs couplés aux protéines G. Le dendrogramme a été produit par la routine pileup du programme GCG (Genetics Computer Group, Madison, WI, USA). L'appartenance des récepteurs olfactifs (en rouge) à une sous-famille de récepteurs couplés aux protéines $G$ apparaît clairement. Cette sous-famille comporte également les récepteurs gustatifs potentiels dont un membre (Gust27) est représenté [37]. Les séquences de récepteurs olfactifs ont été reprises de $[10,11,13,19,20,23,30]$. A titre de comparaison, la sous-famille des récepteurs des amines est présentée sur fond rose. la démonstration fonctionnelle de la nature olfactive de ces récepteurs n'a été démontrée que pour un seul d'entre eux. Raming el al. ont exprimé le récepteur (OR5 dans la lignée cellulaire d'insecte Sf9, en utilisant le baculovirus comme vecteur [30]. Ils ont ainsi pu, sur des préparations membranaires, induire une synthèse d'IP $_{3}$ à la suite de la stimulation par uII mélange de substances odorantes à spectre large (lyral, lilial). De nombreux essais d'expression fonctionnelle dans d'autres systèmes de récepteurs dont les gènes ont été clonés se sont jusqu'à présent soldés par des échecs. Il n'est donc pas établi si les autres récepteurs dont on a cloné les gènes sont tous couplés à cette même cascade intracellulaire $\left(\mathrm{IP}_{3}\right)$. Dans l'hypothèse où aucuu de ces récepteurs ne serait couplé à l'autre cascade impliquée dans l'olfaction (AMPc), il faudrait postuler l'existence d'une deuxième famille de récepteurs olfactifs structurellement distincte. Des récepteurs olfactifs fonctionnels ont également été exprimés dans des ovocytes de xénope, par micro-injection d'ARN messager extrait de muqueuse olfactive de rat ou de poisson-chat, produisant l'activation de canaux chlorure dépendants dı calcium à la suite de la stimulation par la pyrazine (ARNm de rat) ou la I-alanine (ARNm de poisson-chat) [31]. Ia difficulté relative d'obtenir une réponse fonctionnelle d'un récepteur après expression de son gène peut être expliquée partiellement par le très grand nombre de ligands potentiels. Cependant, les récepteurs olfactifs sont généralement considérés comme relativement peu spécifiques, répondant à des substances chimiques variées, et présentant des recouvrements importants de leurs profils respectifs [32]. Il est également possible que des facteurs protéiques additiommels soient nécessaires à l'expression fonctionnelle dans des systèmes hétérologues. Ces facteurs pourraient inclure des protéines extracellulaires telles que les odorant-binding proteins [15], ou des protéines intracellulaires spécifiques impliquées dans la transduction du signal, comme suggéré pour les récepteurs du FMI.P et du C.5a [33]. Tous les récepteurs olfactifs clonés jusqu'à présent possèdent un site potentiel de glycosylation dans leur domaine $\mathrm{N}$-terminal extracellulaire (figure 2A). Certains récepteurs possèdent d'autres sites de glycosylation dans ce domaine N-terminal, ou dans les deuxième ou troisième boucles extracellulaires. La glycosylation des récepteurs olfactifs semble être importante, soit pour la liaison des ligands, soit pour la transduction du signal, car les lectines, telles la concanavaline A ou l'agglutinine de germe de blé, diminuent sensiblement les réponses AMPc et $\mathrm{IP}_{3}$ induites par les odorants [34].

Des cystéines, conservées dans presque toutes les séquences, sont présentes dans les première et seconde boucles extracellulaires. Des cystéines localisées à des positions équivalentes dans les récepteurs adrénergiques et les rhodopsines sont commues pour former un pont disulfure qui stabilise la structure tridimensionnelle du récepteur [35]. Une cystéine conservéc est également présente dans la région C-terminale intracellulaire de certains récepteurs, dans une position identique à la cystéine 341 du récepteur $\beta 2$-adrénergique. Il a été démontré que dans ce dernier récepteur, cette cystcine est palmitoylée, et est responsable de l'ancrage du domaine C-terminal dans la membrane plasmique [35].

Comme dans la transduction des signaux visuels et hormonaux, la phosphorylation des récepteurs semble jouer un rôle primordial dans les mécanismes de terminaison du signal [35]. Il a été montré que l'inhibition de la kinase dépendante de l'AMPc augmente la durée de la réponse (augmentation de l'AMPc intracellulaire) à des odorants tels le citralva. De la même façon, les inhibiteurs de la protéine kinase (; induisent une augmentation prolongée d'IP ${ }_{3}$ en réponse à d'autres odorants [3]. Des sites de phosphorylation potentiels qui pourraient être responsables de cette régulation fonctionnelle sont présents dans tous les récepteurs dont on a cloné les gènes. Presque tous ces récepteurs possèdent dans leur domaine C-terminal un site potentiel pour la protéine kinase C. C.ertains récepteurs possèdent d'autres sites pour la PKC: dans les deuxième et troisième boucles intracellulaires, ainsi qu'un 
site potentiel pour la protéine kinase dépendante de l'AMPc (figure 2A). Il est connu que la phosphorylation de l'extrémité C-terminale - riche en sérine et thréonines - du récepteur $\beta 2$-adrénergique relaie la désensibilisation homologue [35]. L'extrémité C-terminale des récepteurs olfactifs n'est pas particulièrement riche en sérines et thréonines, mais la troisième boucle intracellulaire possède deux à cinq acides aminés qui pourraient constituer la cible de la phosphorylation par les kinases apparentées à la $\beta A R K$. La présence de $\beta A R K_{\text {, }}$ et de $\beta$ Arrestin $_{\text {r }}$ (mais non de $\beta \mathrm{ARK}_{1}$ et $\beta$ Arrestin, $)^{2}$ dans les neurones olfactifs a été mise en évidence récemment. L'inhibition de la désensibilisation homologue par des anticorps dirigés contre ces deux protéines a de plus démontré leur rôle dans ce mécanisme de désensibilisation [36].

\section{Conclusions et perspectives}

Le clonage de la famille de gènes codant pour les récepteurs olfactifs représente un grand pas vers la compréhension de la physiologie de l'olfaction. Néanmoins, nombre de questions restent encore sans réponse concernant la corrélation entre ces récepteurs et les aspects fonctionnels de l'olfaction. Une réponse physiologique a bien été obtenue pour un membre de cette famille, après expression du récepteur recombinant dans un système hétérologue, mais cette caractérisation fonctionnelle devra être étendue à d'autres récepteurs clonés. Des estimations plus précises du nombre de gènes impliqués dans la perception olfactive, de même que l'organisation détaillée de ces gènes dans le génome de différentes espèces de vertébrés vont vraisemblablement être disponibles dans un avenir proche. Ces données, couplées aux études de distribution de récepteurs individuels dans les neurones de la muqueuse olfactive, constitueront la base d'études plus complexes abordant les problèmes du contrôle spatial de l'expression de chacun de ces gènes, de l'établissement des projections des neurones olfactifs dans le bulbe olfactif au cours du développement et des phénomènes de régénération, et de l'évolution de cette famille génique

\section{Summary}

Molecular genetics of olf actory receptors

The understanding of the molecular mechanisms underlying olfactory perception has made considerable progress over the recent years. Key proteins involved in the olfactory signal transduction have been cloned, including the GTPbinding protein $\mathrm{G}_{\text {orf }}$ the olfactoryspecific type III adenylyl cyclase, and the nucleotide-gated olfactory ion channel. The gene family encoding olfactory receptors was further cloned, by low stringency polymerase chain reaction, from rat, human, dog, mouse and catfish, on the basis of their structural similarities with other $G$ protein-coupled receptors. The genes encoding olfactory receptors represent a huge family with up to a thousand members expected in mammalian species and up to a hundred in catfish. The olf actory receptors exhibit all landmarks of G-protein coupled receptors, including the seven putative transmembrane segments, and structural characteristics make them constitute a well identified subfamily. Olfactory receptors display an unusual variability in the sequence of transmembrane segments that are generally conserved between receptor subtypes, sugges- ting a positive selection for mutations occuring in the ligand binding domains. For one of the cloned receptor, the olfactory nature was proven by functional expression of the recombinant protein in a baculovirus system, and stimulation of the $\mathrm{IP}_{3}$ intracellular cascade. All receptors cloned to date have a putative glycosylation site in the $\mathrm{N}$ terminal extracellular domain, and potential phosphorylation sites for protein kinase C, cAMP-dependent protein kinase and $\beta$ ARK related kinases, that are believed to mediate the functional regulation of receptor function by homologous and heterologous desensitization. As a rule, olf actory receptors genes are expressed solely in olfactory mucosa neurons, although some members of this gene family are expressed mainly in testis, with little or no expression in olfactory mucosa. The potential relation of this finding with sperm chemoattraction remains to be elucidated. The genes encoding olfactory receptors seem to be grouped as a limited number of clusters in the human genome. Two of these clusters were identified on chromosomes 17 and 19.

\section{Remerciements}

Nous remercions J.E. Dumont pour son continuel support. Le travail réalisé dans le laboratoire des auteurs a été supporté par le programme belge des Pôles d'Attraction Interuniversitaires impulsé par le Gouvernement Belge, Service du Premier Ministre, Département de la Programmation Scientifique, ainsi que par le Fonds de la Recherche Scientifique Médicale, et Boerhinger Ingelheim. La responsabilité scientifique est assurée par les auteurs. M.P. et F.L.., et P.V. sont respectivement Chercheurs Qualifiés et Aspirant du Fonds National de la Recherche Scientifique. 